\title{
Ultra-high aspect ratio copper nanowires as transparent conductive electrodes for dye sensitized solar cells
}

\author{
Zhaozhao Zhu ${ }^{\dagger}$, Trent Mankowski ${ }^{\dagger}$, Ali Sehpar Shikoh ${ }^{\ddagger}$, Farid Touati ${ }^{\ddagger}$, \\ Mohieddine A. Benammar ${ }^{\ddagger}$, Masud Mansuripur ${ }^{\dagger}$, and Charles M. Falco ${ }^{\dagger}$ \\ ${ }^{\dagger}$ College of Optical Sciences, The University of Arizona, Tucson, USA \\ Department of Electrical Engineering, Qatar University, Doha, Qatar
}

Keywords: copper nanowires, nano-materials, dye-sensitized solar cells, transparent conductive electrodes

\begin{abstract}
We report the synthesis of ultra-high aspect ratio copper nanowires (CuNW) and fabrication of CuNW-based transparent conductive electrodes (TCE) with high optical transmittance (>80\%) and excellent sheet resistance $\left(R_{s}<30 \Omega / \mathrm{sq}\right)$. These CuNW TCEs are subsequently hybridized with aluminum-doped zinc oxide (AZO) thin-film coatings, or platinum thinfilm coatings, or nickel thin-film coatings. Our hybrid transparent electrodes can replace indium tin oxide (ITO) films in dye-sensitized solar cells (DSSCs) as either anodes or cathodes. We highlight the challenges of integrating bare CuNWs into DSSCs, and demonstrate that hybridization renders the solar cell integrations feasible. The CuNW/AZO-based DSSCs have reasonably good open-circuit voltage $\left(V_{o c}=720 \mathrm{mV}\right)$ and short-circuit current-density $\left(J_{s c}=0.96 \mathrm{~mA} / \mathrm{cm}^{2}\right)$, which are comparable to what is obtained with an ITO-based DSSC fabricated with a similar process. Our CuNW-Ni based DSSCs exhibit a good open-circuit voltage $\left(V_{o c}=782 \mathrm{mV}\right)$ and a decent short-circuit current $\left(J_{s c}=3.96 \mathrm{~mA} / \mathrm{cm}^{2}\right)$, with roughly $1.5 \%$ optical-to-electrical conversion efficiency.
\end{abstract}

\section{INTRODUCTION}

Transparent conductive electrodes (TCEs) are essential components of a wide range of opto-electronic devices, especially for many emerging photovoltaic cells. For instance, organic solar cells $(\mathrm{OSCs})^{[1-3]}$, dye-sensitized solar cells $(\mathrm{DSSCs})^{[4-9]}$, and the rapidly improving perovskite solar cells ${ }^{[10,11]}$ all require a transparent conductive window layer to function properly. These devices are usually constructed atop transparent conductive electrodes by depositing the main active layer and additional functional layers. TCEs can be fabricated from many different materials, for instance, metal oxides including indium tin oxide (ITO) ${ }^{[12]}$, aluminum-doped zinc oxide $(\mathrm{AZO})^{[13,14]}$, conductive polymers such as poly(3,4-ethylenedioxythiophene) polystyrene sulfonate (PEDOT: PSS ${ }^{[15]}$, patterned metal grids ${ }^{[16]}$, or randomlyaligned metal nanowire networks ${ }^{[17,18]}$. Among such materials, ITO has been the most successful for commercial applications, due to its excellent optical and electrical characteristics.

TCEs based on metal nanowires, specifically silver nanowires (AgNWs) and copper nanowires (CuNWs), have shown enormous potential with their comparable conductivity and optical transmittance vis-à-vis ITO $^{[17,18]}$. Many researchers have synthesized metallic nanowires via different methods and integrated them into a variety of functional devices, thus demonstrating their potential for practical applications. Compared to silver and indium, copper is more abundant, and the cost of mass-producing copper nanowires is significantly lower than that associated with alternative electrode materials. Additionally, copper has similar bulk resistivity to silver, making it an outstanding conductor. The advantages of copper have driven researchers to investigate the synthesis and optimization of copper nanowires. Rathmell et al ${ }^{[19]}$ demonstrated a solution synthesis of CuNWs, but their nanowire aspect ratio was not ideal compared to that of AgNWs. In 2013, Guo et al demonstrated a different route to synthesis of high aspect-ratio copper nanowires via a solution process $^{[20]}$. Although the synthetic processes of CuNWs have been reproduced many times, hurdles to practical applications of CuNW-based TCEs in real devices are not often discussed in the literature. In several instances, it has been reported that CuNWs easily degrade in their electrical performance ${ }^{[21]}$. Meanwhile, compatibility of CuNWs with various device architectures remains to be determined. More specifically, few published papers have discussed the integration of $\mathrm{CuNW}$ electrodes into dye-sensitized solar cells.

Dye-sensitized solar cells (DSSCs) were introduced by Gratzel in $1991^{[22]}$. The device uses natural or synthetic dyes to sensitize a semiconductor material, which typically consists of titanium dioxide $\left(\mathrm{TiO}_{2}\right)$ nano-particles. In a typical DSSC, the device is usually constructed on a fluorine-doped tin oxide (FTO) or ITO-coated substrate, with the $\mathrm{TiO}_{2}$ layer

Thin Films for Solar and Energy Technology VIII, edited by Michael J. Heben,

Mowafak M. Al-Jassim, Proc. of SPIE Vol. 9936, 993603 · — 2016 SPIE

CCC code: 0277-786X/16/\$18 - doi: 10.1117/12.2237406 


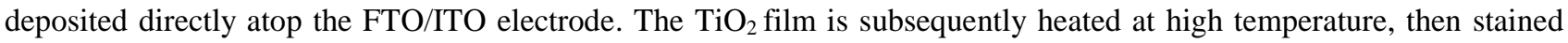
with a natural or synthetic dye. The DSSC is assembled by sandwiching the anode and the cathode (usually platinumcoated ITO) with a thin spacer in between the two electrodes. Finally, a liquid electrolyte fills in the gap between the two electrodes to complete the solar cell. Figure 1 is a schematic illustration of a DSSC. Since their discovery in 1991, DSSCs have drawn much attention due to their low-cost and ease of fabrication. However, as mentioned earlier, there has not appeared in the open literature a detailed analysis of their compatibility with the emerging metal-nanowire-based TCEs. This will be the subject of the present paper.

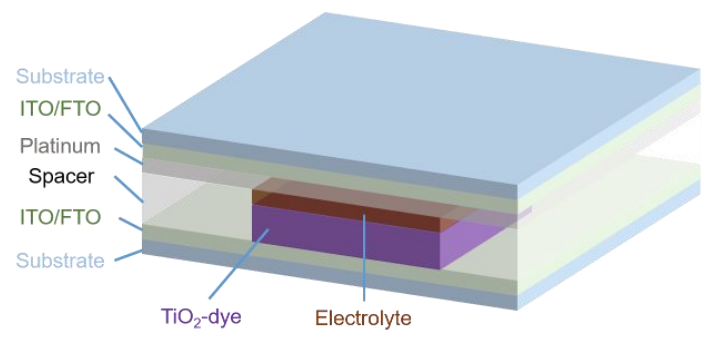

Figure 1. Schematic illustration of a dye-sensitized solar cell.

The particular fabrication process described above has several potential compatibility issues with metal nanowire based TCEs. First, the nanowire-to-substrate adhesion is not strong enough to withstand the forces exerted on the nanowires while depositing the $\mathrm{TiO}_{2}$ layer over the nanowire network. Second, nanomaterials (e.g., copper nanowires) typically have a lower melting temperature than their bulk counterparts, thereby limiting the range of annealing temperatures needed for sintering the $\mathrm{TiO}_{2}$ layer. Last but not least, the liquid electrolyte can corrode the metal electrodes rapidly, leading to device failure.

Although the nanowire TCEs face many challenges with regard to their integration into a DSSC, we believe CuNWbased TCEs share the characteristics of cost-effectiveness and ease-of-fabrication with DSSCs. Therefore, we hybridized our CuNW TCEs with other materials such as AZO thin-film coatings, platinum thin-film coatings, and nickel coatings. Subsequently, we integrated these hybrid electrodes into fully-assembled DSSCs. The hybridization has made it possible for CuNW-based TCEs to be used in DSSCs as either anodes or cathodes, opening possibilities for the large-scale fabrication of fully-functional low-cost devices in the near future.

\section{EXPERIMENTS}

\section{Synthesis of CuNWs}

The solution process of CuNW synthesis is adopted from Guo et $\mathrm{al}^{[20]}$ with slight modifications. Details of the synthesis are described in our previous work ${ }^{[23]}$.To fabricate a CuNW TCE, CuNW solution was used for spray-coating a $1^{\prime \prime} \times 1^{\prime \prime}$ glass substrate. Prior to deposition, the glass substrate was cleaned with methanol, acetone, and deionized water. During the spraying process, the substrate was affixed to a hot-plate maintained at $60{ }^{\circ} \mathrm{C}$. The heated substrate facilitated solvent evaporation. The spraying distance was optimized at $10 \mathrm{~cm}$ for homogeneous nanowire distribution.

After spray-coating deposition of the nanowires on the glass substrate, the samples were placed in a plasma cleaner and exposed to low pressure air plasma $(<5$ Torr) for 2 minutes. This process removes the organic coatings on nanowire surfaces and restores the electrical conductivity of the nanowire thin film.

\section{Deposition of aluminum-doped zinc oxide thin films}

Once the CuNW thin films are exposed to plasma treatment, they are ready for spin-coating aluminum-doped zinc oxide. To prepare the precursors, equimolar mixtures of diethanolamine (DEA) and zinc acetate are dissolved in 2methoxyethanol at a concentration of $0.5 \mathrm{M}$. Subsequently, $\mathrm{Al}\left(\mathrm{NO}_{3}\right)_{3}$ is added to the solution at 2 atomic percent (2 at.\%) to $\mathrm{Zn}^{2+}$ (e.g. $[\mathrm{Al}] /[\mathrm{Al}+\mathrm{Zn}]=2 \%$ ), and the solution is stirred for 1 hour. The precursor solution is aged for at least 24 hours before spin-coating. For each sample, $0.4 \mathrm{~mL}$ of precursor solution was pipetted onto the substrate, and the substrate was spun at $2000 \mathrm{rpm}$ for 40 seconds. Subsequent to spin-coating, the samples were heated at moderate temperatures for 1 hour. 


\section{Pt-coated CuNW thin films}

For direct deposition of platinum, our plasma-treated CuNW thin film samples were mounted on a flat SEM stub. For optimized nanowire surface coverage, the CuNW samples were mounted on $45^{\circ}$ stubs. After sputtering a thin layer of platinum, the mount was rotated and a second layer of platinum was sputtered on the sample. This process was repeated until a thin coating of platinum covered all the exposed sides of the CuNWs.

\section{Ni-coated CuNW thin films}

The Ni-coating process is adopted from Rathmell et al ${ }^{[25]}$ with some modification. Plasma-treated CuNW samples were placed in a beaker with $3 \mathrm{~mL}$ ethylene glycol. Nickel acetylacetonate $(0.1 \mathrm{M}, 0.01 \mathrm{~mL})$ and $\mathrm{NaOH}(0.03 \mathrm{M}, 0.03 \mathrm{~mL})$ were added to the solution while keeping the temperature at $100^{\circ} \mathrm{C}$. Finally, hydrazine solution $(35 \mathrm{wt} \%, 0.157 \mathrm{~mL})$ was added to the mixture. The Ni-coated CuNWs were taken out of the solution after 5 minutes and rinsed with IPA and dried with Nitrogen gas.

\section{Fabrication of dye-sensitized solar cells}

For anodes containing $\mathrm{CuNWs}, \mathrm{TiO}_{2}$ nanoparticles (P25, Sigma-Aldrich) were transferred into a glass vial and heated at $500{ }^{\circ} \mathrm{C}$ in an oven. During the heating process, the nano-particles undergo the color change "white-yellow-white", which is also seen in their thin-film form while annealing. The heated P25 nanoparticles were then dispersed in ethanol (4:7) to form the 'paste', which is subsequently used to deposit on the CuNW/AZO TCEs via doctor blade coating.

For ITO-based anodes, conductive ITO glass slides purchased from NEWView electronics were used $\left(R_{s}=7 \Omega / \mathrm{sq}\right.$ and $T$ $=77 \%$ ). Non-heated P25 nanoparticles were dispersed in ethanol (4:7) to form the paste for blade coating. After the $\mathrm{TiO}_{2}$ mesoporous layer was deposited on the ITO, the anode was heated on a hot-plate at $400{ }^{\circ} \mathrm{C}$ for 15 minutes.

The anodes (either ITO-based or CuNW-based) were then immersed in a staining chamber which contained $0.5 \mathrm{mM}$ cisdiisothiocyanato-bis(2,2'-bipyridyl-4,4'-dicarboxylato) ruthenium(II) bis(tetrabutylammonium) dye (also known as 'N719') in ethanol. The dyeing process typically lasted several hours.

In order to assemble the DSSC, a $0.13 \mathrm{~mm}$ thick square spacer is placed between the two electrodes. Once the anode and cathode are aligned, the EL-HPE high performance electrolyte (791482, Sigma-Aldrich) is filled into the spacer to complete the fabrication process of the solar cell.

\section{Characterization}

The sheet resistance of the electrodes was measured using the 4-probe method with a Keithley 2400 source-meter. Optical transmittance spectra were measured using a Cary-3000 UV-Vis spectrometer and corrected with blank substrates as baselines. Scanning Electron Microscope (SEM) images were taken using a Hitachi 4800 SEM. Grazing angle SEM images were taken by mounting the samples vertically and tilting the mounting stage by $5^{\circ}$. $\mathrm{The}^{\mathrm{TiO}}{ }_{2} \mathrm{meso}^{-}$ porous thin film thickness was measured with a Dektak profilometer. The I-V measurements of the fabricated solar cells were conducted under AM 1.5 solar illumination with a solar simulator, and the data collected with a Keithley 2400 source-meter with customized LabVIEW program.

\section{RESULTS AND DISCUSSION}

As demonstrated in previous work ${ }^{[23]}$, our CuNWs synthesized using the aforementioned solution process have an average length of $70 \mu \mathrm{m}$ and an average diameter of $45 \mathrm{~nm}$. The aspect ratio (length/diameter) of the CuNWs is $\sim 1600$, which is superior to many other CuNWs reported ${ }^{[19]}$. To fabricate the bare CuNW TCE, the nanowires were spray-coated onto glass substrates and subsequently exposed to low-pressure air plasma. The purpose of plasma treatment is to weld the junctions between individual nanowires, and also to remove the organic residues coating the nanowires ${ }^{[23]}$. After the appropriate treatment, our best bare CuNW films on a glass substrate have sheet resistance $R_{s}=22.4 \Omega / \mathrm{sq}$ and optical transmittance (at $550 \mathrm{~nm}$ wavelength) $T=88 \%$. By depositing varying amounts of CuNW, a broad range of sheet resistance and optical transmittance can be obtained, with lower sheet resistance typically associated with lower optical transmittance.

In order to integrate the CuNW-based TCEs into dye-sensitized solar cells (DSSCs), a few obstacles must be overcome. First, to incorporate CuNWs into the anode of the solar cell, the mesoporous $\mathrm{TiO}_{2}$ layer has to be deposited atop the CuNW thin film. The adhesion of CuNWs to the glass substrate is usually not strong enough to withstand the lateral forces exerted on the nanowires, irrespective of whether spin-coating or doctor blade coating is used to deposit the $\mathrm{TiO}_{2}$ 
layer. Second, after the $\mathrm{TiO}_{2}$ film is deposited over the CuNW thin film, a heating process is required, which exceeds the melting point of the nano-sized copper materials. Third, regardless of which electrode (i.e., anode or cathode) the CuNW TCE is used for, the liquid electrolyte corrodes the CuNWs and degrades their electrical performance.

When CuNW TCEs are integrated as anodes into DSSCs, the nanowire-to-substrate adhesion must be strong enough to withstand scotch tape peel tests, and also withstand the exerted lateral forces while applying the $\mathrm{TiO}_{2}$ meso-porous layer. In Fig.2(a), it is seen that, for a bare CuNW sample, nanowires on the right half of the sample have been completely removed after the scotch tape peel test (where the yellow box is drawn). A subsequent sheet resistance measurement confirms that the right-half of the sample is indeed devoid of CuNWs. In our previous work, it was demonstrated that, when an aluminum-doped zinc oxide (AZO) thin film is deposited on the CuNW film and then properly treated, adhesion to the substrate is significantly improved ${ }^{[24]}$. When meso-porous $\mathrm{TiO}_{2}$ is deposited directly on a bare CuNW film, using either spin-coating or doctor blade coating, the CuNWs are ripped off from the substrate, rendering the TCE non-conductive. In contrast, our CuNW/AZO TCEs are mechanically stable, allowing the deposition and staining of the $\mathrm{TiO}_{2}$ layer. Figure 2(b) shows the sheet resistance of a CuNW/AZO sample after five attempts of scotch tape peel; the sample clearly exhibits much improved adhesion to the substrate. The inset in Fig.2(b) is a photograph of a complete CuNW/AZO anode sample stained with N719 overnight; the blade-coated $\mathrm{TiO}_{2}$ layer has an average thickness of $4.8 \mu \mathrm{m}$. After staining, the sheet resistance $R_{s}$ of the CuNW/AZO film slightly increases to $\sim 30 \Omega$ /sq. Figures 2 (c) and 2 (d) are SEM images of a CuNW/AZO film on glass substrate, viewed from two different perspectives. It is seen in both the topdown and grazing views that the CuNW network is fully embedded within the AZO cover layer, thus enhancing the adhesion strength of the TCE to its substrate.
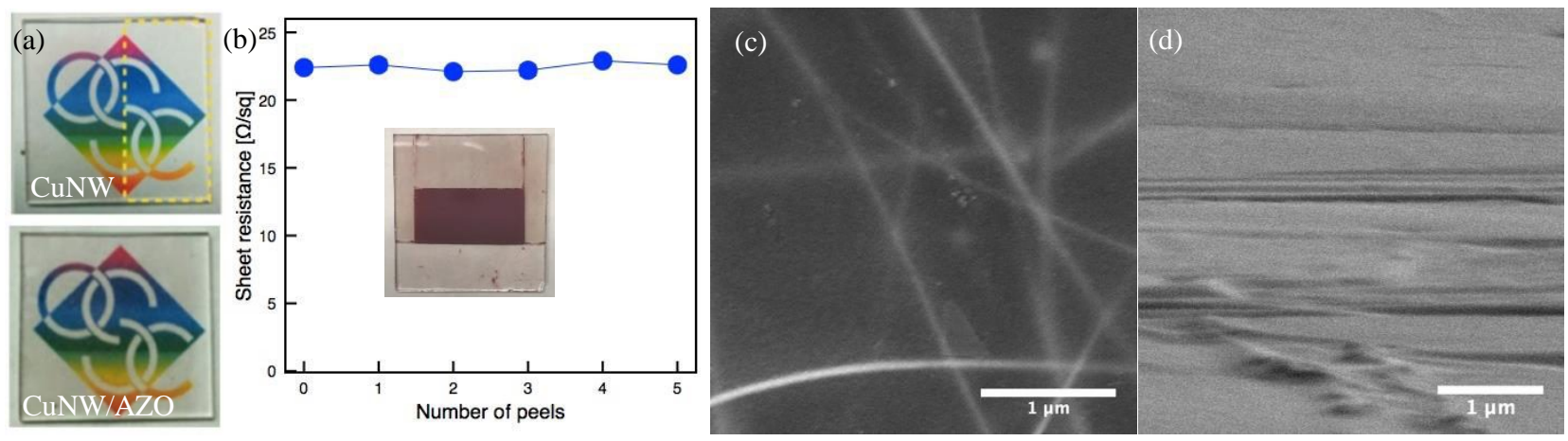

Figure 2. (a) Photographs of bare CuNW (top) on a glass substrate, and CuNW/AZO (bottom) on a glass substrate after a scotch tape peel. The yellow box on the bare CuNW sample indicates the area where the tape was applied. (b) Sheet resistance of a CuNW/AZO sample versus the number of scotch tape peel tests. The inset shows a photograph of a CuNW/AZO sample with meso-porous $\mathrm{TiO}_{2}$ deposited and stained with dye in its central region. (c) Top-down SEM image of a CuNW/AZO sample. (d) Grazing angle SEM image of a CuNW/AZO sample. The nanowires are seen to have been fully embedded within the AZO thin film.
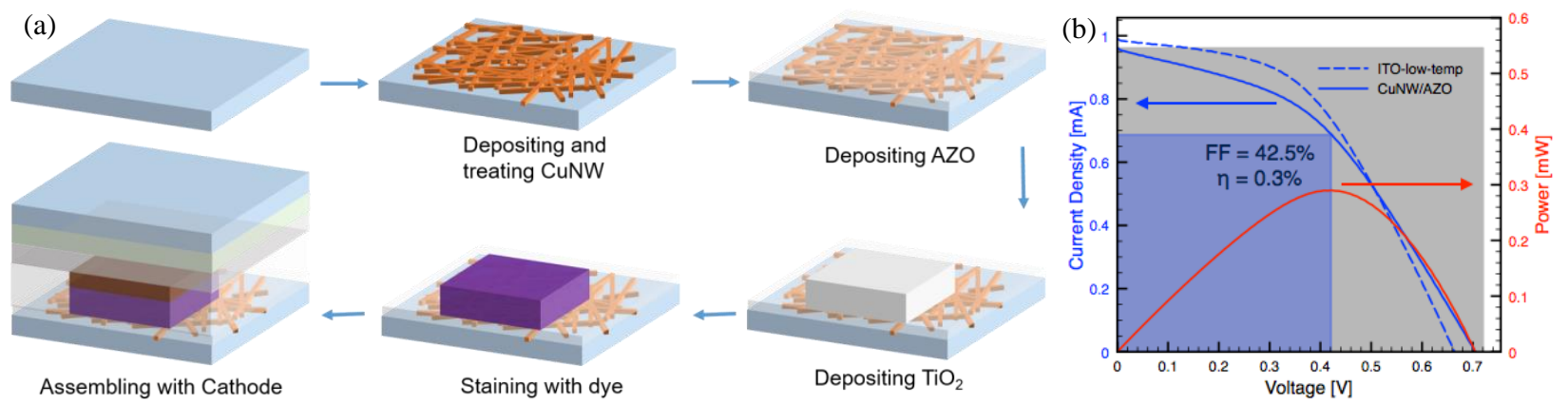

Figure 3. (a) Schematic illustration of the CuNW/AZO dye-sensitized solar cell (DSSC) fabrication process. (b) Measured current-voltage (I-V) characteristic of our CuNW/AZO based DSSC. The measurements were taken under AM 1.5 illumination. 
A number of complete DSSCs were fabricated incorporating the CuNW/AZO anodes. The fabrication process and cell structure are shown in Fig.3(a). Within a series of fabricated cells, we have measured the best open-circuit voltage $\left(V_{o c}\right)$ of $720 \mathrm{mV}$, and short-circuit current density $\left(J_{s c}\right)$ of $0.96 \mathrm{~mA} / \mathrm{cm}^{2}$; the fill factor of the cell was measured at $42.5 \%$. The control sample consisting of substrate/ITO/low-temperature $\mathrm{TiO}_{2} / \mathrm{N} 719$ dye/electrolyte/Pt-ITO/cover layer had $V_{o c}=678$ $\mathrm{mV}$ and $J_{s c}=0.99 \mathrm{~mA} / \mathrm{cm}^{2}$. The current-voltage (I-V) measurement results for the cells are shown in Fig.3(b). Although the strengthened adhesion of CuNWs to the substrate enabled the subsequent deposition of a $\mathrm{TiO}_{2}$ layer atop the nanowire network, the high-temperature annealing/sintering process was still impossible. Following the low-temperature sintering process, we found that the $\mathrm{TiO}_{2}$ nano-particles were not fully sintered. This might explain the low current output and inadequate fill factor of the resulting solar cells. Despite the lower overall performance of the solar cell, the CuNW/AZO-based cell performance is comparable to that of a low-temperature-processed ITO-based sample. We have thus demonstrated the feasibility of integrating CuNW-based TCEs as anodes within a DSSC, albeit at the lower performance levels associated with the low-temperature annealing of $\mathrm{TiO}_{2}$.

In another scenario, if CuNW-based TCEs are used as cathodes for DSSCs, they will be in direct contact with the liquid electrolyte, which is corrosive to CuNWs. Platinum is a good choice of material to serve not only as a protective layer for the CuNW grid, but also as the catalytic film needed for the solar cell operation. In a first attempt, we deposited the platinum coating layer while the CuNW sample was mounted horizontally in the sputtering chamber. The samples came out just as electrically conductive, but with lowered optical transmittance. We immediately pipetted a drop of the liquid electrolyte on the CuNW/Pt sample to test their compatibility. To our surprise, the CuNWs under the platinum coating were still attacked by the liquid electrolyte and disappeared quickly. It occurred to us that the sub-10 nm Pt thin film may not be adequately covering the CuNW grid, as the diameter of individual CuNWs was around $45 \mathrm{~nm}$. The illustration in Fig.4(a) indicates that the sides of individual CuNWs are still exposed while only the tops are covered by the Pt film. To make a continuous Pt coating that would cover the entire CuNW mesh (especially the sides of individual nanowires), we mounted our CuNW sample on a $45^{\circ}$ tilt mount, and rotated the mount's orientation by $90^{\circ}$ after depositing one layer of platinum, repeating the process for all four sides of the CuNW sample. Figure 4(b) is a 2dimensional illustration of the deposition process. By tilting the substrate as described above, platinum was deposited on all sides of individual nanowires. Thus, by repeatedly tilting the CuNW sample during sputter deposition, complete coverage by the Pt film was achieved. The samples prepared with this technique will be referred to as CuNW/4Pt.

(a)

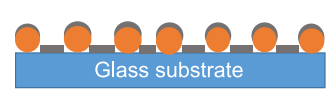

(b)

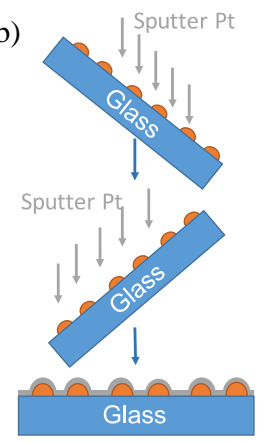

(c)

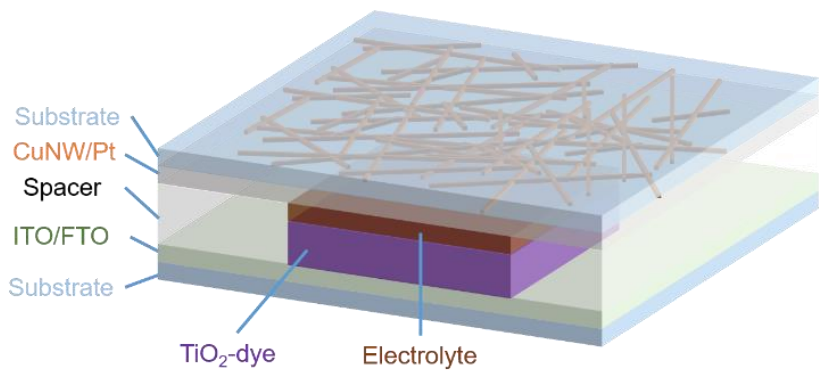

Figure 4. (a) Two dimensional illustration showing that the platinum thin film coating covers only the top portion of the CuNWs. (b) Two-dimensional illustration showing the sputter deposition of a platinum thin film while rocking the substrate, which achieves full coverage on the top as well as on the sides of the CuNWs. (c) Diagram showing a dyesensitized solar cell which incorporates a CuNW/4Pt film as cathode.

The CuNW/4Pt samples were used as transparent cathodes to assemble DSSCs; the device structure diagram can be seen in Fig.4(c). When the cells were illuminated from the back side (cathode-side), we measured $V_{o c}=600 \mathrm{mV}$ and $J_{s c}=0.6$ $\mathrm{mA} / \mathrm{cm}^{2}$. When the cells were illuminated from the ITO side, we measured $V_{o c}=613 \mathrm{mV}$ and $J_{s c}=0.68 \mathrm{~mA} / \mathrm{cm}^{2}$. Although the performance is not ideal for practical applications, we noticed that the life-times of the CuNW/4Pt electrodes had extended to several hours compared to the fairly short life-times of bare CuNW TCEs. The possibility thus exists for using CuNW-based transparent cathodes in fabricating DSSCs.

In addition to sputtering a metallic layer directly onto the CuNW network, one can use a solution process to coat the individual CuNWs of a TCE with a thin metal coating. We adopted and modified an electroless plating method for 
coating our CuNWs with nickel ${ }^{[25]}$. The details of the process are given in our experimental section. Figure 5(a) compares the optical transmittance of the bare CuNW and the Ni-coated CuNW samples. The optical transmittance of the Ni-coated sample is seen to be lower than that of the bare CuNW TCE, but the overall transmittance appears to be more neutral, which can also be seen in the inset photographs. Figure 5(b) shows an SEM image of the Ni-coated CuNW sample, while Fig.5(c) shows the corresponding elemental mapping of nickel in the area depicted in Fig.5(b). It is readily seen that the nickel film is coating only the CuNWs, instead of forming a continuous thin-film on the entire substrate.
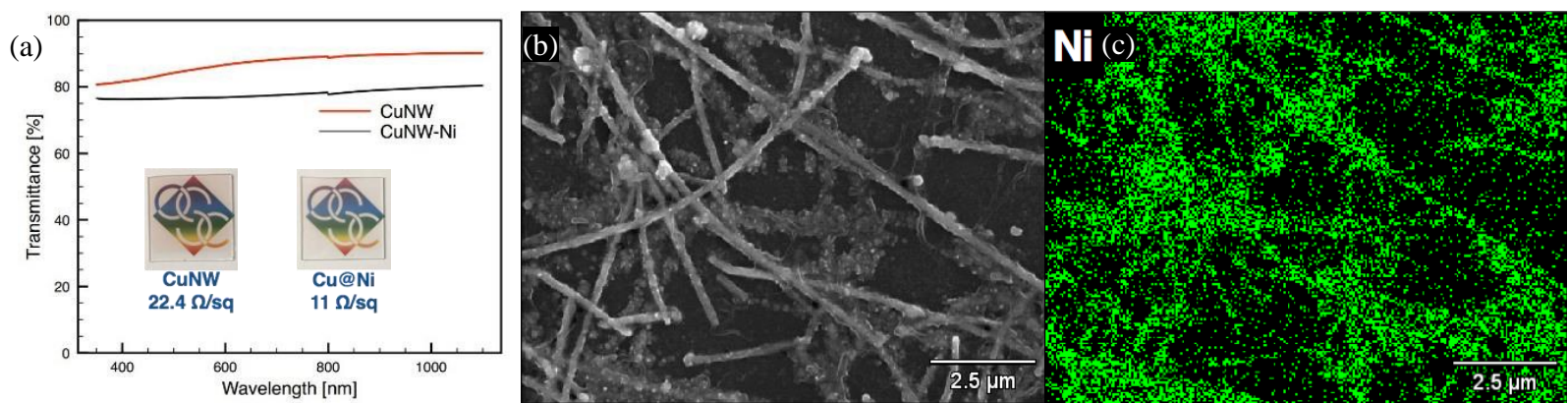

Figure 5. (a) Optical transmittance of bare CuNW and Ni-coated CuNW samples. The inset shows photographs of the two samples placed on OSC logos. (b) SEM image of Ni-coated CuNW sample. (c) Elemental mapping of Ni in the same area as depicted in the SEM image of the sample shown in (b).

The Ni-coated CuNW has enhanced electrical conductivity due to the metal coating; it also shows improved resistance to electrolyte corrosion, making it possible to use it as cathode for a DSSC. Our fabricated DSSC has a high open-circuit voltage $\left(V_{o c}=782 \mathrm{mV}\right)$ and a decent short-circuit current-density $\left(J_{s c}=3.96 \mathrm{~mA} / \mathrm{cm}^{2}\right)$. The fill factor $(49.4 \%)$ of the solar cell is somewhat lower than that of the ITO-based control sample. The overall optical to electrical conversion efficiency of the cell is measured at about $1.5 \%$, which is considerable for a CuNW-based DSSC.

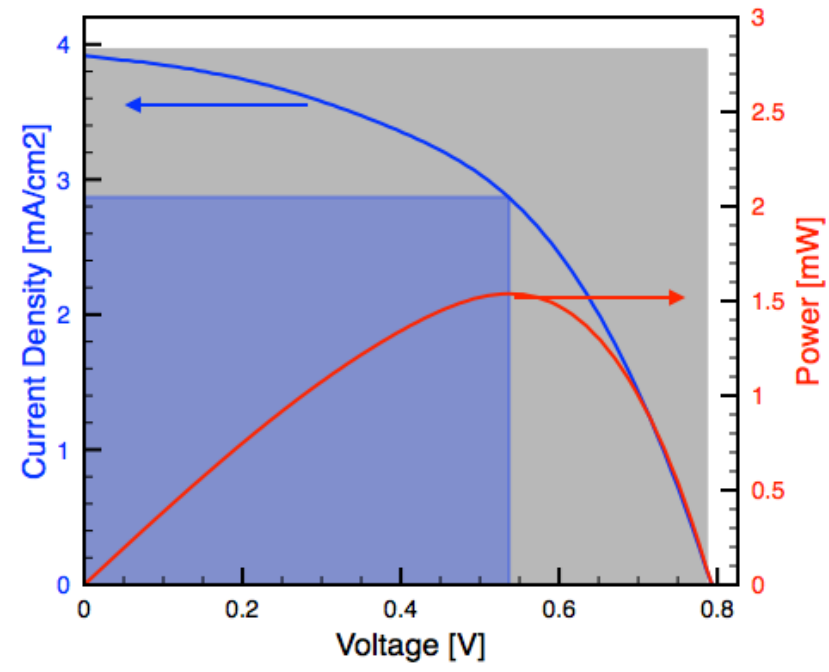

Figure 6. Results of the current-voltage (I-V) measurement of our ITO/TiO2/N719/electrolyte/Ni-coated CuNW dyesensitized solar cell. The measurements were taken under AM 1.5 illumination.

Table 1 summarizes the performance of our fabricated dye-sensitized solar cells that use different electrode configurations. It is seen that CuNW-based TCEs have the potential to replace ITO either as anode or as cathode in a DSSC, but the overall performance is yet to match that of the original ITO-based cells. However, further improvement is expected based on the results of our initial attempts in this direction. Needless to say, replacing ITO with CuNW-based TCEs on both sides of the solar cell promises the manufacturability of ITO-free dye-sensitized solar cells using low-cost copper nano-materials. 
Table 1. Summary of the performance characteristics of various DSSCs fabricated in this work.

\begin{tabular}{|c|c|c|c|c|c|c|}
\hline Anode TCE & $\mathrm{TiO}_{2}$ layer annealing & Cathode & $V_{o c}(\mathrm{mV})$ & $J_{s c}\left(\mathrm{~mA} / \mathrm{cm}^{2}\right)$ & $\mathrm{FF}(\%)$ & $\eta(\%)$ \\
\hline$\overline{\text { ITO }}$ & After deposition & ITO/Pt & 715 & $\overline{5.4}$ & 64.9 & 2.54 \\
\hline ITO & Before deposition & ITO/Pt & 678 & 0.99 & 46.4 & 0.31 \\
\hline CuNW/AZO & Before deposition & ITO/Pt & 720 & 0.96 & 42.5 & 0.96 \\
\hline ITO & After deposition & $\mathrm{CuNW} / \mathrm{Pt}$ & N/A & N/A & N/A & N/A \\
\hline ITO & After deposition & $\mathrm{CuNW} / 4 \mathrm{Pt}$ & 613 & 0.68 & N/A & N/A \\
\hline ITO & After deposition & Ni-coated CuNW & 782 & 3.96 & 49.4 & 1.53 \\
\hline
\end{tabular}

\section{CONCLUSION}

We have synthesized ultra-high aspect ratio CuNWs and hybridized them with several other materials to improve the chemical and/or mechanical stability of the resulting transparent conductive electrodes. These CuNW based TCEs were then integrated into dye-sensitized solar cells, which were fully assembled and then characterized. Our fabricated solar cells incorporating a CuNW/AZO anode exhibit good open-circuit voltage $(720 \mathrm{mV})$, but have a somewhat lower shortcircuit current $\left(0.96 \mathrm{~mA} / \mathrm{cm}^{2}\right)$ compared to all-ITO dye-sensitized solar cells. Our CuNW/4Pt samples show significantly improved resistance to the detrimental effects of the liquid electrolyte, which rapidly corrodes the bare CuNW electrodes. Finally, our Ni-coated CuNW electrodes, which serve as cathodes for dye-sensitized solar cells, exhibit good opencircuit voltage $\left(V_{o c}=782 \mathrm{mV}\right)$ and decent short-circuit current-density $\left(J_{s c}=3.96 \mathrm{~mA} / \mathrm{cm}^{2}\right)$. The hybridization of CuNWs with other materials shows great potential for fabricating ITO-free semi-transparent dye-sensitized solar cells.

\section{ACKNOWLEDGEMENTS}

This work has been made possible by the grant \#5-546-2-222 from the Qatar National Research Fund (a member of the Qatar Foundation). The authors also acknowledge partial support from the Arizona TRIF program. The authors are grateful to Professors N. Peyghambarian and R. Norwood for allowing access to their facilities and instrumentation for parts of the work described in this paper. The statements made herein are solely the responsibility of the authors.

\section{REFERENCE}

[1] K. Lim, S. Jung, J.-K. Kim, J.-W. Kang, J.-H. Kim, S.-H. Choa, and D.-G. Kim, "Flexible PEDOT: PSS/ITO hybrid transparent conducting electrode for organic photovoltaics," Sol Energ Mat Sol C115, 71-78, science direct (2013)

[2] Y.-Y. Choi, S. Kang, H.-K. Kim, W. Choi, and S.-I. Na, "Multilayer graphene films as transparent electrodes for organic photovoltaic devices," Sol Energ Mat Sol C 96, 281-285, science direct (2012)

[3] J.-W. Lim, D.-Y. Cho, Jihoon-Kim, S.-I. Na, and H.-K. Kim, "Simple brush-painting of flexible and transparent Ag nanowire network electrodes as an alternative ITO anode for cost-efficient flexible organic solar cells," Sol Energ Mat Sol C 107, 348-354, science direct (2012)

[4] G. Margulis, G. Christoforo, D. Lam, Z. Beiley, A. Bowring, C. Bailie, A. Salleo, and M. McGehee, "Spray Deposition of Silver Nanowire Electrodes for Semitransparent Solid-State Dye-Sensitized Solar Cells," Adv Energy Mater 3(12), 1657-1663, Wiley (2013)

[5] Grätzel, M., "Dye-sensitized solar cells," J Photochem Photobiology C Photochem Rev 4(2), 145-153, science direct (2003).

[6] Hardin, B., Gaynor, W., Ding, I.-K., Rim, S.-B., Peumans, P.., McGehee, M., "Laminating solution-processed silver nanowire mesh electrodes onto solid-state dye-sensitized solar cells," Org Electron 12(6), 875-879, science direct (2011).

[7] Liu, B.-T.., Wang, Z.-T., "Graphene oxide/poly(3,4-ethylenedioxythiophene):polystyrenesulfonate layers on silver nanowire working electrodes enhance the power conversion efficiencies of dye-sensitized solar cells in a low temperature process," Rsc Adv 6(53), 47185-47191, rsc (2016).

[8] Wang, X., Zhi, L.., Müllen, K., “Transparent, Conductive Graphene Electrodes for Dye-Sensitized Solar Cells,” Nano Lett 8(1), 323-327, acs (2008).

[9] Law, M., Greene, L., Johnson, J., Saykally, R.., Yang, P., "Nanowire dye-sensitized solar cells," Nat Mater 4(6), 455-459, nature (2005).

[10] Batmunkh, M., Shearer, C., Biggs, M.., Shapter, J., "Solution processed graphene structures for perovskite solar cells," J Mater Chem 4(7), 2605-2616, rsc (2016).

[11] You, J., Hong, Z., Yang, Y., Chen, Q., Cai, M., Song, T.-B., Chen, C.-C., Lu, S., Liu, Y., et al., "Low-Temperature SolutionProcessed Perovskite Solar Cells with High Efficiency and Flexibility," ACS Nano 8(2), 140105153751009, acs (2014).

[12] Schulze, K., Maennig, B., Leo, K., Tomita, Y., May, C., Hüpkes, J., Brier, E., Reinold, E.., Bäuerle, P., “Organic solar cells on indium tin oxide and aluminum doped zinc oxide anodes," Appl Phys Lett 91(7), 073521, aip (2007). 
[13] Lee, J.-H.., Park, B.-O., "Transparent conducting ZnO:Al, In and Sn thin films deposited by the sol-gel method," Thin Solid Films 426(1-2), 9499 (2003).

[14] Ohyama, M., Kozuka, H., Yoko, T., "Sol-Gel Preparation of Transparent and Conductive Aluminum-Doped Zinc Oxide Films with Highly Preferential Crystal Orientation," Journal of the American Ceramic Society 81(6), 1622-1632 (1998).

[15] Hong, W., Xu, Y., Lu, G., Li, C., Shi, G., "Transparent graphene/PEDOT-PSS composite films as counter electrodes of dyesensitized solar cells," Electrochemistry Communications 10(10), 1555-1558 (2008).

[16] Zou, J., Yip, H.-L., Hau, S.., Jen, A., "Metal grid/conducting polymer hybrid transparent electrode for inverted polymer solar cells," Appl Phys Lett 96(20), 203301, aip (2010).

[17] Lee, J.-Y., Connor, S., Cui, Y., Peumans, P., "Solution-processed metal nanowire mesh transparent electrodes," Nano letters 8(2), 689-692 (2008).

[18] Chen, Z., Ye, S., Stewart, I.., Wiley, B., "Copper Nanowire Networks with Transparent Oxide Shells That Prevent Oxidation without Reducing Transmittance," ACS Nano 8(9), 9673-9679, acs (2014).

[19] Rathmell, A., Bergin, S., Hua, Y., Li, Z.., Wiley, B., "The Growth Mechanism of Copper Nanowires and Their Properties in Flexible, Transparent Conducting Films," Adv Mater 22(32), 3558-3563, Wiley (2010).

[20] Guo, H., Lin, N., Chen, Y., Wang, Z., Xie, Q., Zheng, T., Gao, N., Li, S., Kang, J., et al., "Copper Nanowires as Fully Transparent Conductive Electrodes," Sci Reports 3, nature (2013).

[21] Kholmanov, I., Domingues, S., Chou, H., Wang, X., Tan, C., Kim, J.-Y., Li, H., Piner, R., Zarbin, A., et al., "Reduced Graphene Oxide/Copper Nanowire Hybrid Films as High-Performance Transparent Electrodes," Acs Nano 7(2), 1811-1816, acs (2013).

[22] B. O’Regan, M. Grätzel, “A low-cost, high-efficiency solar cell based on dye-sensitized colloidal TiO2 films” Nature 335 (1991) 737.

[23] Zhu, Z., Mankowski, T., Balakrishnan, K., Shikoh, A., Touati, F., Benammar, M., Mansuripur, M.., Falco, C., "Ultrahigh Aspect Ratio Copper-Nanowire-Based Hybrid Transparent Conductive Electrodes with PEDOT:PSS and Reduced Graphene Oxide Exhibiting Reduced Surface Roughness and Improved Stability," Acs Appl Mater Interfaces 7(30), 16223-16230, acs (2015).

[24] Zhu, Z., Mankowski, T., Balakrishnan, K., Shikoh, A.S., Touati, F., Benammar, M.A., Mansuripur, M. and Falco, C.M., 2016. "Hybrid transparent conductive electrodes with copper nanowires embedded in a zinc oxide matrix and protected by reduced graphene oxide platelets," Journal of Applied Physics, 119(8), p.085303.

[25] A. Rathmell, M. Nguyen, M. Chi, and B. Wiley, "Synthesis of oxidation-resistant cupronickel nanowires for transparent conducting nanowire networks," Nano Lett. 12(6), 3193-3199, acs (2012). 